\title{
Interfacial bubbles formed by plunging thin liquid films in a pool
}

\author{
Louis Salkin, ${ }^{1}$ Alexandre Schmit, ${ }^{1,2}$ Richard David, ${ }^{1}$ Alexandre \\ Delvert, ${ }^{1}$ Eric Gicquel, ${ }^{1}$ Pascal Panizza, ${ }^{1}$ and Laurent Courbin ${ }^{1}$ \\ ${ }^{1}$ IPR, UMR CNRS 6251, Campus Beaulieu, Université de Rennes 1, 35042 Rennes, France \\ ${ }^{2}$ NANO, UR1268, BIA, INRA, rue de la Géraudière, 44316 Nantes, France
}

\begin{abstract}
We show that the immersion of an horizontally-suspended thin film of liquid in a pool of the same fluid creates an interfacial bubble, that is, a bubble at the liquid-air interface. Varying the fluid properties, the film's size and its immersion velocity, our experiments unveil two formation regimes characterized by either a visco-capillary or an inertio-capillary mechanism that controls the size of a produced bubble. To rationalize these results, we compare the pressure exerted by the air flow under a plunging film with the Laplace pressure needed to generate film dimpling, which subsequently yields air entrapment and the production of a bubble. This physical model explains the power-law variations of the bubble size with the governing dimensionless number for each regime.
\end{abstract}

PACS numbers: 47.55.N- 47.55.Ca 47.55.db

Introduction. Air entrapment is a commonly observed phenomenon in free surface flows ${ }^{1}$. It occurs in both natural phenomena such as falling raindrops $s^{2,3}$ and industrial processes, e.g. coating, ink-jet printing, and spray cooling. The creation of air bubbles is generally detrimental to such processes and their applications in industry. For instance, it often changes for the worse both optical and mechanical properties of final products such as paint or molten glass. For these reasons, air entrapment is an active research topic that has been investigated for a wide variety of flow configurations. Examples of these include drops impacting smooth ${ }^{4-13}$ or rough $^{14-16}$ solids and liquid surfaces ${ }^{17-24}$, solid objects plunged into a pool ${ }^{25-28}$, liquid jets either poured into fluids ${ }^{29-31}$ or impacting solid surfaces ${ }^{32}$, and collapsing interfacial bubbles ${ }^{33,34}$. For all these situations, studies share the common goal of identifying the key variables at play, obtaining a physical understanding of the problem, and predicting the formation of bubbles and their size.

Here, we study an uninvestigated situation where the impact at constant velocity of an horizontal thin film of liquid with a pool of the same fluid produces film dimpling, air entrapment and an interfacial bubble. Our experiments show that the size of such a bubble increases with both the impact velocity and size of a plunging film; by contrast, it does not depend on a film's thickness. Varying the impact speed, we observe two possible formation regimes. The bubble size results from either a viscocapillary (small enough speed) or an inertio-capillary (sufficiently large speed) mechanism. The regime at low speeds bears analogies with drop impact on solids ${ }^{12}$. In contrast with this case however, the evolution of the bubble size with the speed is monotonic for plunging films and we do not observe a maximum bubble size for an intermediate impact speed ${ }^{12}$.

Experiments: setup, materials and methods. As depicted in Fig. 1(a), our setup consists of a thin-liquid film suspended horizontally on a ring (radius $R$ ) mounted on a vertical linear motor stage (Aerotech ACT115DL) that is connected to a controller (Aerotech Soloist HPE). A ring is made of wire with a radius $b=500 \mu \mathrm{m}$ that is negligible compared to $R=10-80 \mathrm{~mm}$. A house-made Labview software controls the motion of a ring upwards or downwards at constant speed $v=10^{-3}-1 \mathrm{~m} \mathrm{~s}^{-1}$ over a distance $d \leq 0.5 \mathrm{~m}$. Imposing a constant speed to a stationary ring or stopping a moving one requires acceleration and deceleration phases, respectively. We impose a constant acceleration $\left(0-50 \mathrm{~m} \mathrm{~s}^{-2}\right)$ during these two phases by selecting the distances $d_{a}$ and $d_{d}$ over which the ring accelerates and decelerates, respectively; the corresponding absolute values of the accelerations are $a_{a}=\frac{v^{2}}{2 d_{a}}$ and $a_{d}=\frac{v^{2}}{2 d_{d}}$ [see Fig. 1(b)].

In our experiments, a ring, which is first immersed in the liquid bath, is withdrawn quasistatically at a height $h_{0}$ from the free surface. As discussed in ${ }^{35}$, because of surface tension, withdrawing the ring creates a minimal surface of revolution, i.e., a catenoid. Above a critical height $h_{c} \approx 0.66 R<h_{0}$, this shape becomes unstable and collapses to leave a planar film on the ring and an interfacial bubble on the bath ${ }^{36}$. This bubble is then punctured with a needle to remove it from the free surface. We measure the thickness $(e=1-10 \mu \mathrm{m})$ of the planar film with a spectrometer (Avantes AvaSpec-2048). This film is then moved downward so that it impacts the free surface at constant velocity $v$ (see Fig. 1). A certain amount of air (density $\rho_{a}$ and dynamic viscosity $\eta_{a}$ ) is trapped between film and liquid surface upon impact, creating an interfacial bubble of radius $R_{b}$, as illustrated by the series of high-speed images in Fig. 1(c).

The liquid is either a soap solution (2 wt\% Fairy (P\&G) and 98 wt\% water) or polydimethylsiloxane (PDMS) (Sylgard 184, Dow Corning). Both fluids are Newtonian and their dynamic viscosities $\eta_{\ell}$ measured at $20{ }^{\circ} \mathrm{C}$ with an Anton Paar MCR 301 rheometer are 1 $\mathrm{mPa} \mathrm{s}$ (soap solution) and 6.4 Pa s (PDMS). $\rho_{\ell}$ herein denotes the liquid density: $\rho_{\ell}=965 \mathrm{~kg} \mathrm{~m}^{-3}$ (PDMS) and $\rho_{\ell}=1000 \mathrm{~kg} \mathrm{~m}^{-3}$ (soap solution). We use pendant drop 


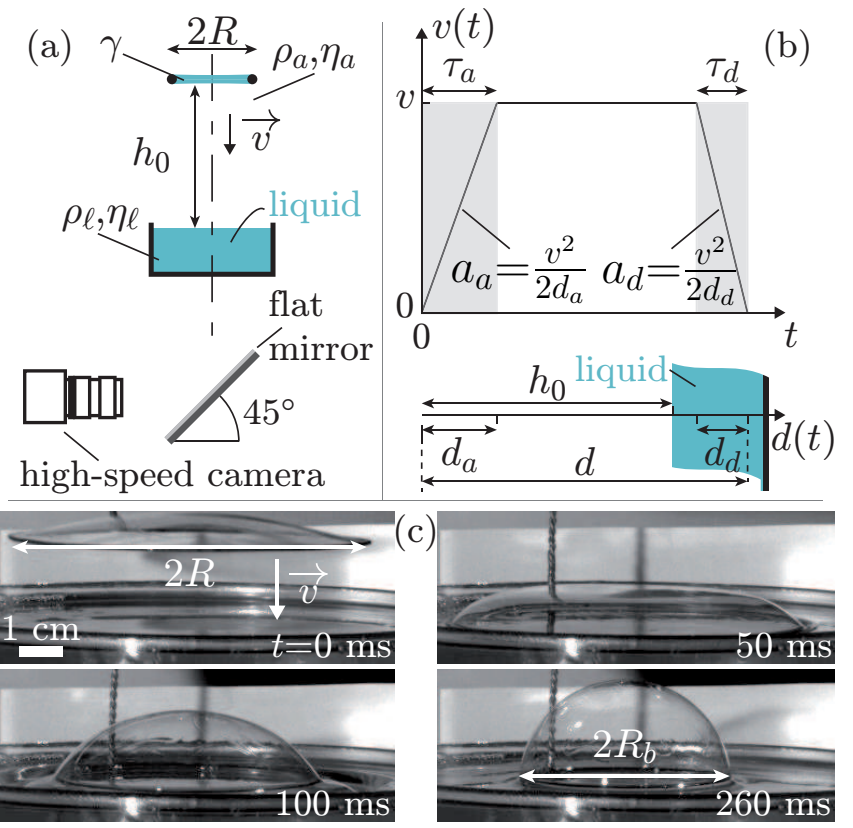

FIG. 1. Schematics of (a) the setup and (b) the temporal evolution of the impact speed defining the experimental variables. The shaded areas in (b) indicate acceleration and deceleration phases. (c) Side-view images illustrating the creation of a bubble for $v=0.4 \mathrm{~m} \mathrm{~s}^{-1}, R=41 \mathrm{~mm}$ and $h_{0}=95 \mathrm{~mm}$.

tensiometry (Teclis Tracker) to determine the liquid-air surface tension $\gamma$. We find $25 \mathrm{mN} \mathrm{m}^{-1}$ (soap solution) and $20 \mathrm{mN} \mathrm{m}^{-1}$ (PDMS). To measure a bubble's radius, we record its formation from below with a high-speed camera (Photron SA3) working at 250-2000 frames s ${ }^{-1}$.

Oscillations at small heights. We begin by studying the variations of $R_{b}$ with the initial height $h_{0}$ for different impact speeds, all other variables remaining constant (figure 1 defines the variables at play). We select values of $d, d_{a}$ and $d_{d}$ that satisfy the three conditions $d>h_{0}$, $d_{a}<h_{0}$ and $d_{d}<d-h_{0}$ so that $v$ is constant when the ring impacts the liquid pool [see Fig. 1(b)]; $d_{d}$ is smaller than the depth of the pool.

When $v$ is large enough, i.e., larger than about $0.2 \mathrm{~m} \mathrm{~s}^{-1}$ for a medium ring size, the evolution of $R_{b}$ with $h_{0}$ is characterized by damped oscillations for any speed [see Fig. 2(a)]. $R_{b}^{\infty}$ and $\Delta h_{0}$ denote respectively the constant bubble radius found within the limit $h_{0} \rightarrow \infty$ and the distance between two consecutive maxima in the signal $R_{b}$ vs. $h_{0}$. Both quantities increase with $v$ [Fig. 2(a)]. The amplitude of the observed oscillations, i.e. the size of a produced bubble for small $h_{0}$, is in fact a function of the applied acceleration $a_{a}$ (see Fig. S3 and related discussion $\mathrm{in}^{37}$ ).

Side-view videos of plunging films under conditions similar to those of Fig. 2 help to understand the oscillatory signal $R_{b}$ vs. $h_{0}$. For both PDMS and the soap solution, they indeed reveal that a film oscillates vertically as it moves towards the pool $\left(\mathrm{see}^{37}\right.$ for MovieS1
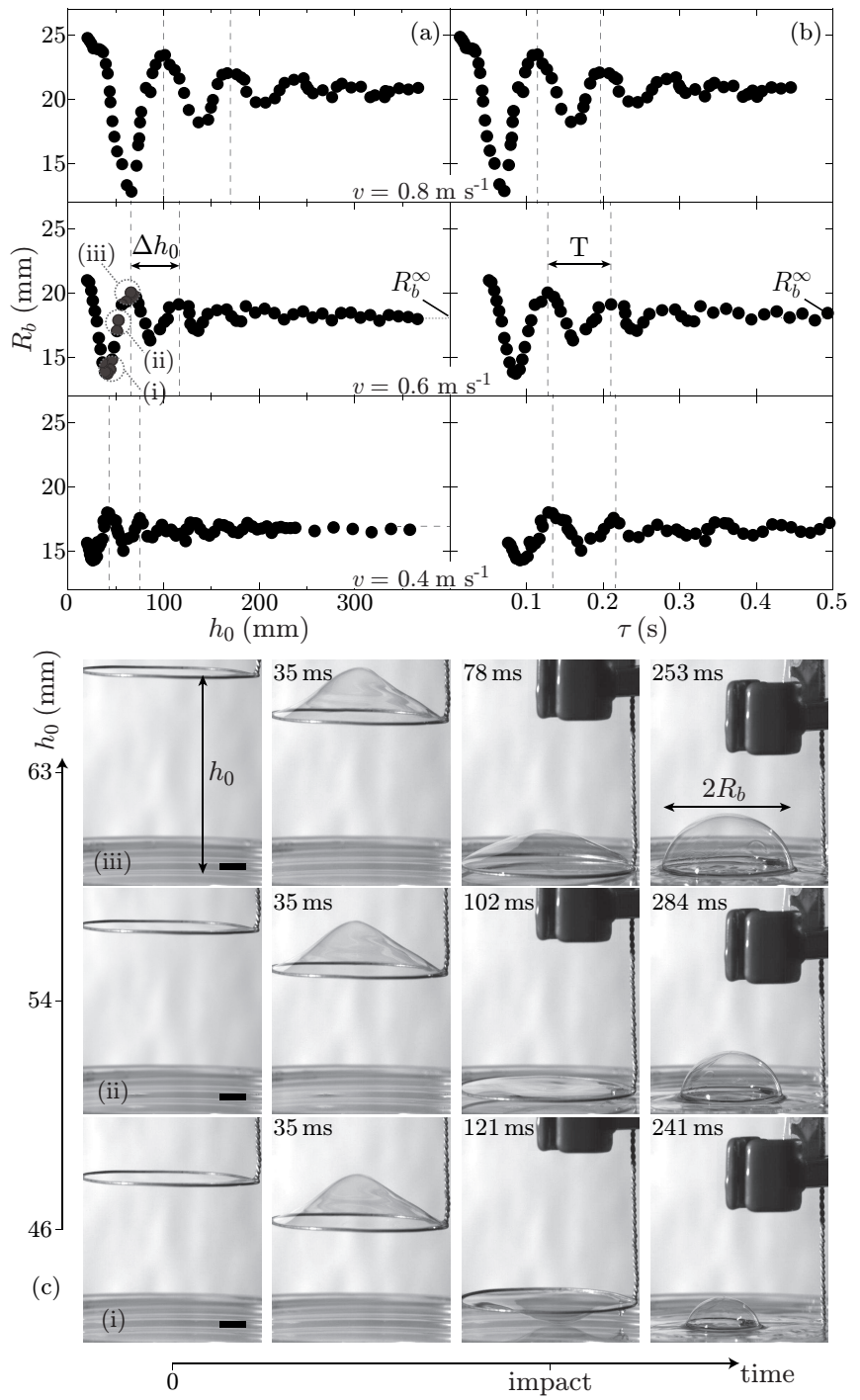

FIG. 2. Radius of the interfacial bubble $R_{b}$ versus (a) height $h_{0}$ and (b) $\tau=\frac{h_{0}-d_{a}}{v}$ for a ring of radius $R=31 \mathrm{~mm}$ and three impact speeds $v$ as indicated. Other parameters are $d_{a}=10 \mathrm{~mm}$ and $d_{d}=8 \mathrm{~mm}$ so that the acceleration $a_{a}$ is (top graph) $32 \mathrm{~m} \mathrm{~s}^{-2}$, (middle graph) $16 \mathrm{~m} \mathrm{~s}^{-2}$ and (bottom graph) $8 \mathrm{~m} \mathrm{~s}^{-2}$. (c) Series of photographs illustrating the influence of the position at impact of an oscillating film on the size of a produced bubble in the case of $v=0.6 \mathrm{~m} \mathrm{~s}^{-1}$ in Fig. 1(a) for three different heights $h_{0}$ as indicated [(i)-(iii)]. Scale bar: $1 \mathrm{~cm}$. The liquid is the soap solution.

and a brief discussion of the oscillations of a film). In what follows, we discuss the forces acting on a film in the non-inertial reference frame of the ring that is accelerated with regard to that of the laboratory. In this reference frame, during the acceleration phase, a fictitious force deflects the film upward from its horizontal equilibrium position, surface tension being a resisting force that opposes this deflection. Air friction also acts on the film and the combination of the three forces give the shape of the film. The fictitious force vanishes when the ring 
reaches a stationary velocity. No longer at equilibrium, the film then relaxes to a new steady state position. This surface-tension driven relaxation induces air friction on the film which causes the damped oscillatory variations of $R_{b}$ with $h_{0}$. When a ring impacts the pool, the volume of entrapped air is maximal (resp. minimal) when the film presents a maximal deflection above (resp. below) the ring. Figure 2(c) shows a series of photographs that illustrate how the position at impact of an oscillating film influences the size of a produced bubble. To validate the above scenario, we plot in Fig. 2(b) the evolution of $R_{b}$ with the time $\tau=\frac{h_{0}-d_{a}}{v}$ elapsed between the end of the acceleration phase and the contact between ring and pool. As indicated by the dashed vertical lines in Fig. 2(b), the distance between two consecutive maxima corresponds to the constant period of oscillations $\mathrm{T}$ that we manually measure using the first two maxima that are easier to define than those found at higher heights. The period $\mathrm{T}$ of the damped oscillations, which does not depend on $v_{c}<v<1 \mathrm{~m} \mathrm{~s}^{-1}$, is an intrinsic parameter of the soap film. We show in ${ }^{37}$ that this time corresponds to the first antisymmetric mode of oscillation of a film ${ }^{38,39}$.

Bubble size in the high height regime. $h_{0}$ is hereafter large enough so that nonoscillating films impact the pool and $R_{b}^{\infty}$ is independent of both $h_{0}$ and $a_{a}$ (see Fig. S3 $\left.i^{37}\right)$. The variations of $R_{b}^{\infty}$ with $v$ for both fluid systems unveil two formation regimes, each characterized by a power-law response (see Fig. 3). As mentioned earlier, in contrast with drop impact on solids ${ }^{12}, R_{b}^{\infty}$ is increasing monotonically with $v$. The exponent of the power law above a threshold speed $v_{c}$ is larger than at small $v$. In addition, the results shown in Fig. 3 suggest that these exponents are independent of both $R$ and the nature of the fluid and that $v_{c}$ decreases with $R$. Also, the curves shown in Fig. 3 tend to flatten out for the largest speeds when $R_{b}^{\infty}$ gets close to $R$.

Discussion and model. Understanding our findings requires to identify the variables controlling the response. As indicated above, the power laws seem to be independent of the liquid properties ${ }^{40}$, i.e., $\rho_{\ell}$ and $\eta_{\ell}$. By contrast, to account for film dimpling, we expect the surface tension $\gamma$ to be a controlling variable. Hence, $R_{b}^{\infty}$ should depend on $v, R, \gamma, \rho_{a}$ and $\eta_{a}$. One could speculate that the film thickness $e$ also plays a role in the formation of bubbles. To characterize the influence of $e$, we have performed experiments with plunging films prepared by withdrawing a ring at different speeds. Because of Frankel's law of film thickness, such films have different thicknesses, in the range $e=1-10 \mu \mathrm{m}$ for our experiments. We have found that the impact of these films with the pool for a fixed speed $v$ produces bubbles having the same radius in both regimes. One could also consider that gravity influences the formation of bubbles. However, in our experiments, gravity $\left(\propto \pi R^{2} e \rho_{\ell} g\right)$ should dominate effects of surface tension $(\propto 2 \pi R \gamma)$ when the films are sufficiently thick, that is, $e>\frac{2 \gamma}{\rho_{\ell} g R}$. This estimate corresponds to films larger than about $100 \mu \mathrm{m}$ which is an order of magnitude larger than the thick-

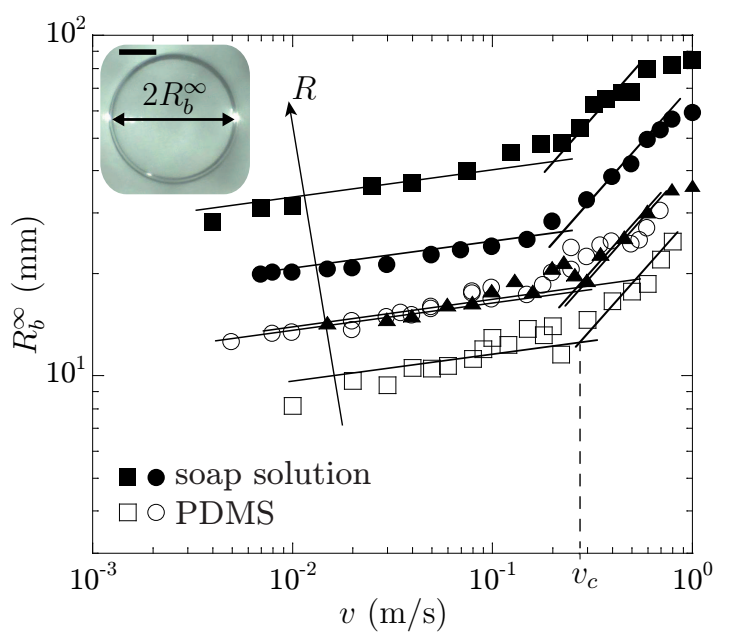

FIG. 3. $R_{b}^{\infty}$ versus $v$ for different radii $R: 82 \mathrm{~mm}(\boldsymbol{\square}), 51 \mathrm{~mm}$ $(\bullet), 36 \mathrm{~mm}(\boldsymbol{\Delta}), 35 \mathrm{~mm}(\circ)$ and $25 \mathrm{~mm}(\square)$. Open and closed symbols stand for PDMS and the soap solution, respectively. For any liquid and value of $R$, one observes a power-law response with a small (resp. large) exponent when $v<v_{c}$ (resp. $v>v_{c}$ ) as indicated by the lines that are guides for the eyes. Inset: bottom-view picture of an interfacial bubble defining the measured bubble radius $R_{b}^{\infty}$. Scale bar: $5 \mathrm{~mm}$.

nesses of the films that we use. Hence, on can consider that surface tension dominates gravity for the whole study. A rapid dimensional analysis then shows that $R_{b}^{\infty} / R$ is a function of the capillary number $\mathcal{C}=\eta_{a} v / \gamma$ and the Weber number $\mathcal{W} e=\rho_{a} v^{2} R / \gamma$, two dimensionless quantities based on gas properties $\left(\rho_{a}\right.$ and $\left.\eta_{a}\right)$ and incorporating surface tension. In other words, this analysis predicts that $R_{b}^{\infty} / R$ varies as $\mathcal{W} e^{\alpha} \mathcal{C}^{\beta}$ where $\alpha$ and $\beta$ are two numerical constants to determine. As demonstrated below, the formation of bubbles is in fact controlled by either a visco-capillary $(\alpha=0)$ or an inertio-capillary $(\beta=0)$ mechanism.

We expect the mechanism comparing gas inertia and surface tension to control the response when the Reynolds numbers $\mathcal{R} e=\mathcal{W} e / \mathcal{C}$ is large. This is the case for the regime seen at speeds above $v_{c}$ in Fig. $3 ; \mathcal{R} e \approx 1000$ for $R=50 \mathrm{~mm}$ and $v=0.3 \mathrm{~m} \mathrm{~s}^{-1}$. When $v>v_{c}$, the film in its reference frame encounters a uniform air flow of constant speed $v$ that creates a dimple of mean curvature $\kappa$ in it. Using Bernoulli's principle, this translates to $\rho_{a} v^{2} / 2=4 \gamma \kappa$ so that $\kappa^{-1}=8 R / \mathcal{W} e$. We assume the dimple to be a spherical cap whose volume is that of the produced bubble. Then, using geometric considerations to write a first-order approximation of the function $R_{b}^{\infty} / R$ around $\mathcal{W} e=0$, one readily finds $R_{b}^{\infty} / R \simeq \frac{3^{1 / 3}}{4} \mathcal{W} e^{1 / 3} ;$ in our experiments, $\mathcal{W} e=10^{-5}-3$. Figure 4 shows that this physical model concurs well with experiments when $v>v_{c}$ as it collapses data onto a single curve and the predicted prefactor $\frac{3^{1 / 3}}{4} \approx 0.36$ is close to the experimental one $(\approx 1)$. Also shown in Fig. 4 and already mentioned when discussing the results reported 


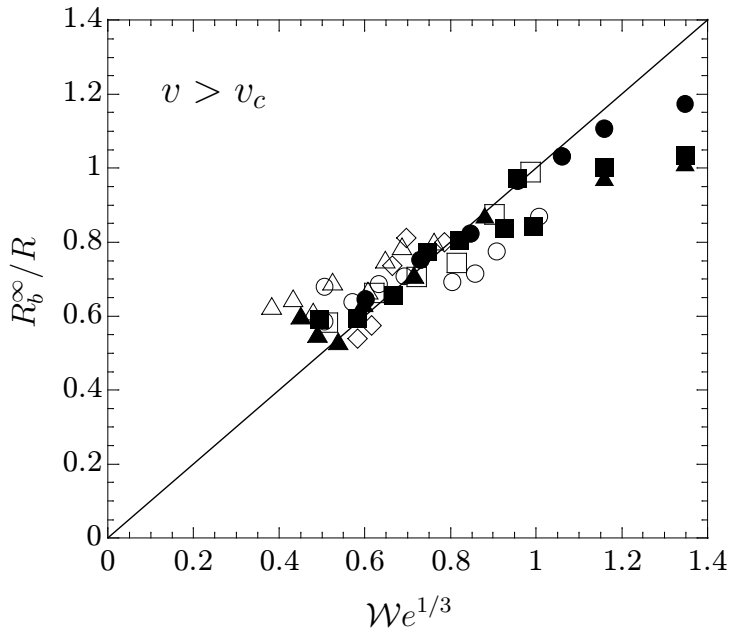

FIG. 4. Variations of $R_{b}^{\infty} / R$ with $\mathcal{W} e^{1 / 3}$ for different values of $R: 36 \mathrm{~mm}(\mathbf{\Delta}), 15 \mathrm{~mm}(\Delta)$ and $10 \mathrm{~mm}(\diamond)$ and as indicated in Fig. $3(\bullet, \square, \circ, \square)$. Open and closed symbols correspond to experiments conducted with PDMS or the soap solution, respectively. The line is a guide for the eyes whose slope is 1 .

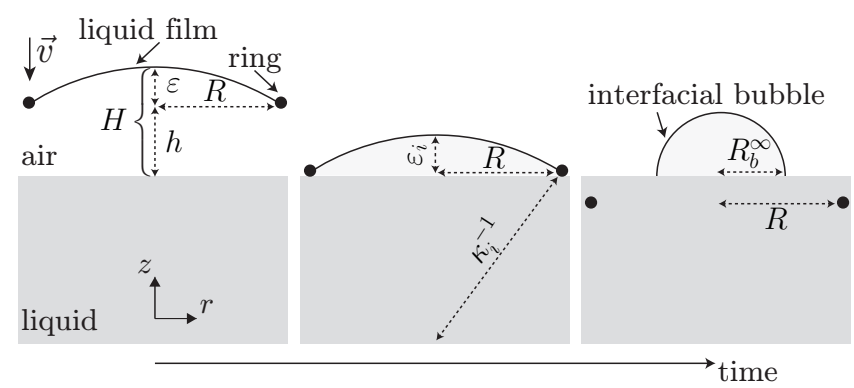

FIG. 5. Schematic (not to scale) of film dimpling as a ring reaches the pool surface. Defined are the geometric variables.

in Fig. 3, when the Weber number is larger than about $1.33, R_{b}^{\infty} / R$ gets near to or larger than 1 , the data flatten out and our model written around $\mathcal{W} e=0$ fails to rationalize experiments.

When $v<v_{c}$, film dimpling originates from the viscous flow of the air squeezed under a film as a ring reaches the pool. One can model this radial flow with a lubrication approximation of the Stokes equation:

$$
\frac{\partial p_{a}(r, t)}{\partial r} \sim \eta_{a} \frac{\partial^{2} u_{r}(r, t)}{\partial z^{2}}
$$

where $u_{r}$ is the radial component of the air velocity, $z$ is the vertical direction and $p_{a}$ is the air pressure (see Fig. 5). The pressure difference across a dimpled film $\Delta p_{a}$ is a function of the film mean curvature $\kappa(r, t)$ :

$$
\Delta p_{a}(r, t)=p_{a}(r, t)-p_{0}=4 \gamma \kappa(r, t)
$$

with $p_{0}$ the atmospheric pressure. $\kappa(r, t)$ and the film's

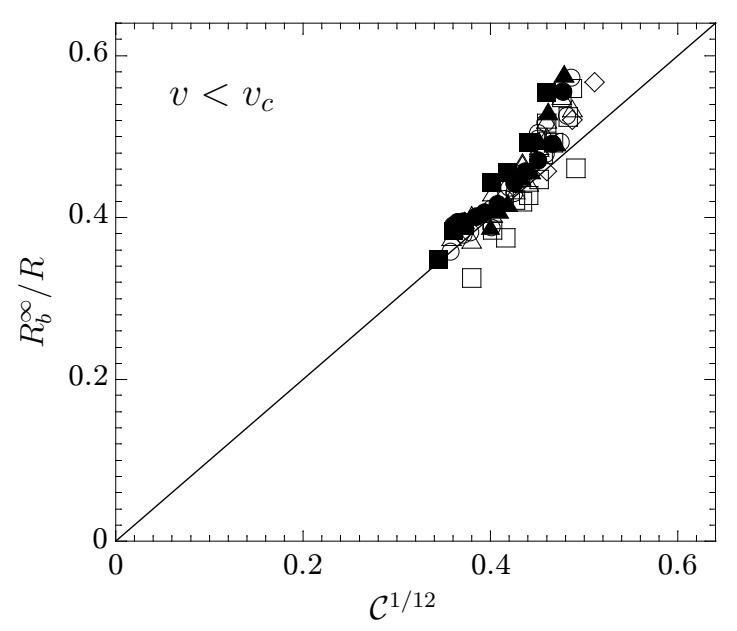

FIG. 6. Shown is $R_{b}^{\infty} / R$ vs. $\mathcal{C}^{1 / 12}$. Symbols are identical to those of Fig. 4. The line with slope 1 is a guide for the eyes.

profile $\varepsilon(r, t)$ (see Fig. 5) are related by the geometry:

$2 \kappa(r, t)=\left[1+\left(\frac{\partial \varepsilon(r, t)}{\partial r}\right)^{2}\right]^{\frac{-3}{2}}\left[\frac{\partial^{2} \varepsilon(r, t)}{\partial r^{2}}+\frac{\frac{\partial \varepsilon(r, t)}{\partial r}\left[1+\left(\frac{\partial \varepsilon(r, t)}{\partial r}\right)^{2}\right]}{r}\right]$.

The lubrication equation describing the gas flow underneath the film closes the problem:

$$
\frac{\partial H(r, t)}{\partial t}=\frac{1}{r} \frac{\partial}{\partial r}\left(\frac{r H(r, t)^{3}}{12 \eta_{a}} \frac{\partial p_{a}(r, t)}{\partial r}\right)
$$

where $H(r, t)=h(t)+\varepsilon(r, t)$ is small compared to the other flow direction, i.e. $H(r, t) \ll R, h(t)$ being the distance between ring and pool at time $t$ (see Fig. 5 ).

Using these basic elements modeling the flow, we now rationalize our findings for $v<v_{c}$ at the level of scaling. An order-of-magnitude analysis of Eq. (1) and mass conservation give $\Delta p_{a} \sim \eta_{a} \frac{R u_{r}}{H^{2}}$ and $u_{r} \sim \frac{v R}{H}$, respectively. Hence, $\Delta p_{a} \sim \eta_{a} \frac{v R^{2}}{H^{3}}$. Assuming the dimple to be a spherical cap of radius $\kappa_{i}^{-1}$ when the ring meets the pool, i.e. when $h=0$, the maximum deflection of the film at impact $\varepsilon_{i} \ll R$ can be written $\varepsilon_{i} \sim R^{2} \kappa_{i}$ (see Fig. 5). The increase of pressure in the gas layer under the film yields film dimpling, whereas capillary forces tend to maintain the film flat. This translates to a balance between pressure in the gas and Laplace pressure, which provides $\varepsilon_{i} \sim R C^{1 / 4}$; as in our experiments $\mathcal{C}^{1 / 4} \approx 0.03-0.18$, our approximation $\varepsilon_{i} \ll R$ is reasonable. From volume conservation between the spherical cap and the created bubble, $\varepsilon_{i} R^{2} \sim R_{b}^{\infty 3}$, we then obtain a prediction for the variations of the normalized bubble size $R_{b}^{\infty} / R \sim \mathcal{C}^{1 / 12}$. As shown in Fig. 6, these physical arguments allow us to understand our results when $v<v_{c}$ as this prediction concurs with experimental data.

Equating the two expressions found for $\frac{R_{b}^{\infty}}{R}$ in the two 


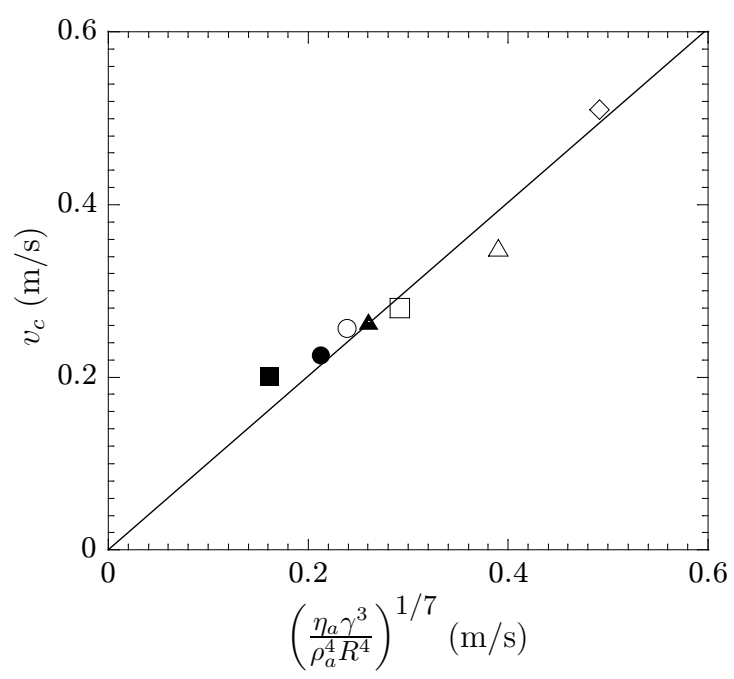

FIG. 7. Comparison between measured and predicted [Eq.(5)] threshold $v_{c}$. Symbols are identical to those of Fig. 4. The line is the best linear fit with y-intercept 0 and slope 1.005 .

formation regimes, one derives the threshold speed:

$$
v_{c} \sim\left(\frac{\eta_{a} \gamma^{3}}{\rho_{a}^{4} R^{4}}\right)^{1 / 7}
$$

which mirrors the measured one (see Fig. 7). Besides the nature of the fluid density in Eq.(5), it may be noted that the expression of $v_{c}$ is similar to that of the speed at which a bubble size is maximum for drop impact on solids ${ }^{12}$. Also, non-dimensionalization of Eq.(5) gives the transition between regimes in terms of dimensionless quantities: the creation of a bubble is controlled by the inertio-capillary mechanism when $\left(\mathcal{W} e^{4} / \mathcal{C}\right)^{1 / 7}>1$ or the visco-capillary mechanism otherwise.

Conclusion. We conclude with three remarks. Firstly, within the limit $v \rightarrow 0$, we expect the formation of a bubble to be solely controlled by geometric variables. Within this quasistatic limit, a film should indeed remain flat enough for the volume of entrapped air to be that of a cylinder having height $b / 2$ and radius $R$. We then anticipate that $R_{b}^{\infty} / R \sim\left(\frac{3 b}{4 R}\right)^{\frac{1}{3}}$; in our experiments, this quasistatic limit bubble radius is in the $R_{b}^{\infty}=3.3-13.4 \mathrm{~mm}$ range. Unfortunately, validating this simple prediction is a difficult task since slight variations in the horizontalness of the ring at very small speeds cause large variations of the measured bubble size. Secondly, for speeds larger than $\sqrt{\frac{8 \gamma}{\rho_{a} R}}\left(1.3-4 \mathrm{~m} \mathrm{~s}^{-1}\right.$ in our experiments), the air dynamic pressure $\frac{\rho_{a} v^{2}}{2}$ overcomes the Laplace pressure $4 \gamma R^{-1}$ required to transform the liquid film into a bubble of radius $R^{41-43}$, bubbles should therefore form. We have not observed this regime because of the limited maximum speed $\left(1 \mathrm{~m} \mathrm{~s}^{-1}\right)$ imposed by our motor stage. Lastly, one might suppose that a bubble could form before a ring reaches a stationary velocity, i.e., during the acceleration phase. This would occur when the dynamic pressure $\frac{\rho_{\ell} v^{2}}{2} \frac{e}{d_{a}}$ caused by the fictitious force acting on an accelerating film overcomes the threshold in capillary pressure $4 \gamma R^{-1}$. Hence, bubbles should be produced during the acceleration phase for speeds larger than $\sqrt{\frac{8 \gamma}{\rho_{a} R}} \sqrt{\frac{\rho_{a}}{\rho_{\ell}} \frac{d_{a}}{e}}$; interestingly, in contrast with bubbles formed by blowing on soap films ${ }^{42,43}$, it is worth noting that this threshold speed for making bubbles is a function of the film thickness. Since in our experiments this threshold speed is in the range $1.3-12 \mathrm{~m} \mathrm{~s}^{-1}$, we have not observed the creation of bubbles during the acceleration phase either. To validate the predicted thresholds at high speeds, measurements should be conducted for speeds larger than the maximum speed of our motor which would require developing a new experimental setup. Designing such a setup would be a challenging task since it should offer the same specifications as our vertical linear motor stage (precise control of the speed of a moving frame, the acceleration needed to reach this velocity and the deceleration required to stop the moving frame) so that previous data can be compared to new ones.

Acknowledgement. We thank A. Saint-Jalmes for helping us with viscosity and surface tension measurements and J. H. Snoeijer for fruitful discussions.
1 A. L. Yarin, Drop impact dynamics: splashing, spreading, receding, bouncing..., Annu. Rev. Fluid Mech. 38, 159-192 (2006).

2 D. C. Blanchard and A. H. Woodcock, Bubble formation and modification in the sea and its meteorological significance, Tellus 9, 145-158 (1957).

3 A. Prosperetti, L. A. Crum, and H. C. Pumphrey, The underwater noise of rain, J. Geophys. Res. 94, 3255-3259 (1989).

${ }^{4}$ S. Chandra and C. T. Avedisian, On the collision of a droplet with a solid surface, Proc. R. Soc. Lond. A 432, 13-41 (1991).

${ }^{5}$ M. Rein, Phenomena of liquid drop impact on solid and liquid surfaces, Fluid Dyn. Res. 12, 61-93 (1993).

${ }^{6}$ S. T. Thoroddsen, T. G. Etoh, K. Takehara, N. Ootsuka, and Y. Hatsuki, The air bubble entrapped under a drop impacting on a solid surface, J. Fluid Mech. 545, 203-212 (2005)

7 P. D. Hicks and R. Purvis, Air cushioning and bubble entrapment in three-dimensional droplet impacts, J. Fluid Mech. 649, 135-163 (2010)

8 S. Mandre, M. Mani, and M. P. Brenner, Precursors to splashing of liquid droplets on a solid surface, Phys. Rev. Lett. 102, 134502 (2009).

9 M. M. Driscoll and S. R. Nagel, Ultrafast interference imaging of air in splashing dynamics, Phys. Rev. Lett. 107, 
154502 (2011).

10 J. M. Kolinski, S. M. Rubinstein, S. Mandre, M. P. Brenner, D. A. Weitz, and L. Mahadevan, Skating on a film of air: drops impacting on a surface, Phys. Rev. Lett. 108, 074503 (2012).

11 S. Mandre and M. P. Brenner, The mechanism of a splash on a dry solid surface, J. Fluid Mech. 690, 148-172 (2012).

${ }^{12}$ W. Bouwhuis, R. C. A van der Veen, T. Tran, D. L. Keij, K. G.Winkels, I. R. Peters, D. Van der Meer, C. Sun, J. H. Snoeijer, and D. Lohse, Maximal air bubble entrainment at liquid-drop impact, Phys. Rev. Lett. 109, 264501 (2012).

13 R. C. A. van der Veen, T. Tran, D. Lohse, and C. Sun, Direct measurements of air layer profiles under impacting droplets using high-speed color interferometry, Phys. Rev. E 85, 026315 (2012).

${ }^{14}$ L. Courbin, J. C. Bird, M. Reyssat, and H. A. Stone, Dynamics of wetting: from inertial spreading to viscous imbibition, J. Phys.: Condens. Matter 21, 464127 (2009).

15 P. Tsai, R. C. A. van der Veen, M. van de Raa, and D. Lohse, How micropatterns and air pressure affect splashing on surfaces, Langmuir 26, 16090-16095 (2010).

16 R. C. A. van der Veen, M H. W. Hendrix, T. Tran, P. A. Tsai, and D. Lohse, How microstructures affect air film dynamics prior to drop impact, Soft Matter 10, 3703-3707 (2014).

17 L. Esmailizadeh and R. Mesler, Bubble entrainment with drops, J. Colloid Interface Sci. 110, 561-574 (1986).

${ }^{18}$ H. N. Oguz and A. Prosperetti, Bubble entrainment by the impact of drops on liquid surfaces, J. Fluid Mech. 219, 143-179 (1990).

19 S. T. Thoroddsen, T. G. Etoh, and K. Takehara, Air entrapment under an impacting drop, J. Fluid Mech. 478, 125-134 (2003).

${ }^{20}$ M. M. Driscoll, C. S. Stevens, and S. R. Nagel, Thin film formation during splashing of viscous liquids, Phys. Rev. E 82, 036302 (2010).

${ }^{21}$ Q. Deng, A. V. Anilkumar, and T. G. Wang, The role of viscosity and surface tension in bubble entrapment during drop impact onto a deep liquid pool, J. Fluid Mech. 578, 119-138 (2010).

${ }^{22}$ M.-J. Thoraval and S. T. Thoroddsen, Contraction of an air disk caught between two different liquids, Phys. Rev. E 88, 061001(R) (2013)

23 D. L. Keij, K. G.Winkels, H. Castelijns, M. Riepen, and J. H. Snoeijer, Bubble formation during the collision of a sessile drop with a meniscus, Phys. Fluids 25, 082005 (2013).

24 T. Tran, H. de Maleprade, C. Sun, and D. Lohse, Air entrainment during impact of droplets on liquid surfaces, J. Fuid Mech. 726, R3 (2013).

25 R. Bergmann, D. van der Meer, S. Gekle, A. van der Bos, and D. Lohse, Controlled impact of a disk on a water surface: cavity dynamics, J. Fluid. Mech. 633, 381-409
(2009).

26 J. O. Marston, I. U. Vakarelski, and S. T. Thoroddsen, Bubble entrapment during sphere impact onto quiescent liquid surfaces, J. Fluid Mech. 680, 660-670 (2011).

27 A. Marchand, T. S. Chan, J. H. Snoeijer, and B. Andreotti, Air entrainment by contact lines of a solid plate plunged into a viscous fluid, Phys. Rev. Lett. 108, 204501 (2012).

28 P. D. Hicks, E. V. Ermanyuk, N. V. Gavrilov, and R. Purvis, Air trapping at impact of a rigid sphere onto a liquid, J. Fluid Mech. 695, 310-320 (2012).

29 Y. Zhu, H. N. Oguz, and A. Prosperetti, On the mechanism of air entrainment by liquid jets at a free surface, J. Fluid. Mech. 404, 151-177 (2000).

30 J. Eggers, Air entrainment through free-surface cusps, Phys. Rev. Lett. 86, 4290 (2001).

31 E. Lorenceau, D. Quéré, and J. Eggers, Air entrainment by a viscous jet plunging into a bath, Phys. Rev. Lett. 93, 254501 (2004).

32 B. Pouligny and M. Chassande-Mottin, Air ingestion by a buckled viscous jet of silicone oil impacting the free surface of the same liquid, Phys. Rev. Lett. 100, 154501 (2008).

33 An interfacial bubble is a bubble at a liquid-air or solid-air interface.

34 J. C. Bird, R. de Ruiter, L. Courbin, and H. A. Stone, Daughter bubble cascades produced by folding of ruptured thin films, Nature 465, 759-762 (2010).

35 L. Salkin, A. Schmit, P. Panizza, and L. Courbin, Influence of boundary conditions on the existence and stability of minimal surfaces of revolution made of soap films, Am. J. Phys 219, 839-847 (2014).

36 L. Salkin, A. Schmit, P. Panizza, and L. Courbin, (unpublished).

37 See Supplemental Material at [] for a movie illustrating the generation of interfacial bubbles and the vertical oscillations of a film during its motion, a brief discussion about the oscillation of a film, the characteristic time of the damped oscillatory variations of the bubble size and the effects of the initial acceleration $a_{a}$ on the oscillations.

38 Y. Couder, J. M. Chomaz, and M. Rabaud, On the hydrodynamics of soap films, Physica D 37, 384 (1989).

39 V. O. Afenchenko, A. B. Ezersky, S. V. Kiyashko, M. I. Rabinovich, and P. D. Weidman, The generation of twodimensional vortices by transverse oscillation of a soap film, Phys. Fluids 10, 390-399 (1998).

${ }^{40}$ We have no conclusive evidence concerning the dependence on $\rho_{\ell}$ as the two liquids used in our experiments have similar densities.

41 H. Bouasse, Capillarité - Phénomènes Superficiels (Librairie Delagrave, Paris, 1924).

42 L. Salkin, A. Schmit, P. Panizza, and L. Courbin, Generating soap bubbles by blowing on soap films, Phys. Rev. Lett. 116, 077801 (2016).

${ }^{43}$ P. Panizza and L. Courbin, Bubble blowing by the numbers, Phys. Today 69(7), 78 (2016). 